\title{
STRATÉGIE DE RÉSEAU : UNE SOURCE DE DÉVELOPPEMENT ET DE PERFORMANCE POUR LES ENTREPRISES EN SITUATION DE RISQUES PROHIBITIFS
}

\author{
Boualem ALIOUAT ${ }^{*}$, Hadj NEKKA ${ }^{* *} \&$ Zahir YANAT ${ }^{* * *}$
}

\begin{abstract}
Résumé - Observant que les stratégies de réseaux ne s'inscrivent pas formellement dans la culture stratégique de certaines entreprises, notamment au Maghreb, quel qu'en soit l'étendue (entre entreprises appartenant à une même région, à un pays ou à une échelle internationale), la thèse soutenue dans cet article considère au contraire que cette forme inter-organisationnelle serait une source première du développement et de performance pour ces entreprises en situation de risques qui occasionne des coûts de transaction prohibitifs. Cette recherche exploratoire consiste dans un premier temps à mettre l'accent sur les difficultés qui ne permettent pas à des entreprises, ici le cas des firmes algériennes, de se développer en réseaux et de discuter ensuite les raisons qui favorisent davantage les partenariats publics-privés. L'exploitation de l'ensemble de ces informations nous permet de proposer quelques recommandations pour inciter les entreprises, notamment algériennes, à tendre davantage vers la forme en réseau pour se développer dans un avenir au sein d'un environnement hostile porteur de risques prohibitifs. Ces propositions sont transposables à toutes formes d'environnements hostiles au sens économique du terme.
\end{abstract}

Mots clés : réseaux ; performance ; environnement hostile ; entreprises algériennes ; clusters.

\section{Introduction}

De manière générale, l'amplification de la complexité des processus de connaissance à la base de toute innovation contraint désormais les entreprises à aller au-delà de leurs frontières pour compléter ou parfaire leurs propres capacités innovatrices (Becker et Dietz, 2004). De même, la compression des cycles de vie des technologies oblige les entreprises à

\footnotetext{
* Université Nice Sophia Antipolis, GREDEG, 250 rue Albert Einstein - Bâtiment 2, 06560 Valbonne, Boualem.Aliouat@unice.fr

** Université d'Angers, GRANEM, 4 boulevard Lavoisier, 49016 Angers cedex, hadj.nekka@univ-angers.fr

*** KEDGE Business School, 680, cours de la Libération, 33405 Talence cedex - France, Zahir.Yanat@bem.edu
} 
revoir leurs investissements d'innovation et à élargir leurs assises technologiques sur des bases collaboratives (Nijssen et al., 2001). La coopération peut ici à la fois contribuer à un développement plus rapide des innovations, améliorer l'accès aux marchés à l'échelle mondiale, permettre de bénéficier d'économies d'échelles, et rendre possible un partage des coûts et une répartition des risques (López, 2008 ; De Faria et al., 2010). Elle permet aussi d'établir des combinaisons complémentaires et des synergies susceptibles de réduire les contraintes et d'optimiser la vélocité d'intégration des capacités dynamiques (Becker et Dietz, 2004 ; Dachs et al., 2008). La décision de coopérer signifie alors que les partenaires mettent à disposition des unes et des autres leurs options et gammes technologiques (Mowery et al., 1998 ; Caloghirou et al., 2003). Ainsi, les entreprises impliquées ont plus de connaissances à leur disposition que celles qui agissent isolément (Gomes-Casseres et al., 2006 ; Rodrigues Bandeira et al., 2012). Cela explique en partie qu'à partir des années 1980, les alliances stratégiques et les développements en réseaux ont connu une inflation croissante au sein des pays industrialisés et des pays émergents sous l'influence convergente de l'ouverture des marchés et des accords de libre-échange.

Les pays en transition ne sont pas en reste. Notamment, lorsque l'adaptation à des environnements en carence de régulation ou de gouvernance génère un contexte de risques « pays " qui entraîne des coûts de transaction prohibitifs pour leurs entreprises (Aliouat, 2014a). Certains de ces pays concernés proposent aujourd'hui des politiques volontaristes et vont saisir des opportunités institutionnelles offertes par la Banque mondiale ou le FMI pour se restructurer sur la base de pôles d'innovations et de politiques d'industrialisation en réseau. Ainsi, les premières initiatives concrètes en matière de technopôles et de réseaux d'innovation en région MENA datent de la fin des années 1990. Le pôle El Ghazala près de Tunis, le MUCSAT près d'Alexandrie, Berytech à Beyrouth, le Techno-park et le Casashore de Casablanca, le smart village près du Caire, le CyberParc de Sidi Abdallah près d'Alger, Techonopolis de Rabat ou Tanger Med, le 10ème plan tunisien des 6 technopôles, les pôles de Bizerte, de Borj Cedria, de Sfax, de Bouimane, de Fez ou le futur Technopôle d'Agadir constituent des enjeux importants dans les stratégies métropolitaines de croissance économique, de développement et de compétitivité durable basée sur l'innovation en réseau. Même si aucune initiative commune maghrébine n'est à répertorier. Algérie, Maroc et Tunisie se développent de manière séparée et disparate (Aliouat, 2014b). Dans cet ensemble territorial, l'Algérie, qui nous intéresse tout particulièrement ici, affiche un certain retard dans le développement de l'innovation et de l'industrialisation en réseau, en partie en raison de ses spécificités économiques et socio-historiques. L'approche dite "spatiale » démontre que le vecteur déterminant en matière de bifurcation vers une trajectoire soutenue de développement est d'ordre institutionnel. Elle concerne la capacité décisionnelle des entités régionales à exploiter les leviers de la mondialisation et de la multi-localisation des chaines de valeur industrielles. Le développement singulier des régions périphériques du Sun Belt américain qui s'étend de la Californie (Silicon Valley) à la Caroline-du-Nord, en passant par Dallas (Texas) ou Boston, la Floride ou Austin, présente des traits communs avec l'émergence de pôles mondiaux de compétitivité technologique localisés dans des régions périphériques comme Nice Sophia Antipolis (France), Aerospace Valley à Toulouse, Kista Science City à Stockholm, Anvers pour le diamant, Peterborough, au Royaume-Uni, pour les biens et services environnementaux, Silicon Fen à Cambridge (GB), Helsinki et Turku en Finlande, la Communauté Autonome du Pays Basque (Espagne), Multimedia cluster Hilversum aux Pays-Bas, Munich ou Heidelberg (Allemagne), Parme (Italie), Singapour, 
Hong Kong, ou Osaka. Ces pôles forment une toile ou un réseau ouvert à toute entité territoriale qui dispose d'une capacité autonome à intégrer les réseaux mondiaux en raison de son efficacité stratégique à faire valoir des compétences distinctives dans un climat d'affaires incitatif. Une attention particulière est accordée à la localisation territoriale et à l'attrait des environnements comme étant des indicateurs du « degré de clustering ». À partir d'une analyse du contexte économique maghrébin et d'une analyse de l'essence des réseaux, nous proposons ici une réflexion sur le degré de clustering de l'Algérie et ses chances de développement économique en réseau industriel et technologique.

\section{Contexte de développement et de performance au Maghreb}

Le développement des pays du Maghreb engage des problématiques et des niveaux de risques majeurs pour les deux décennies à venir. Il faut rappeler que le taux de croissance moyen durant la période 2007-2013 a été de 0,9\% pour les pays européens alors qu'il a dépassé $5 \%$ pour les pays du sud de la zone méditerranée dont fait partie l'Algérie. Malgré cela, ces mêmes pays du sud ont des taux de chômage qui oscillent entre 23,6 et 40\%, ainsi que des facteurs de compétitivité assez faibles. Cela nous amène à rappeler que même si ces pays $\mathrm{du}$ sud sont parfois dans la croissance, ils ne sont pas forcément dans le développement ${ }^{1}$. Le problème, c'est que les perspectives de croissance se dégraderont brutalement à partir de 20202. La moyenne de croissance du PIB passe de 5.3\% en 2010 à $6.8 \%$ en 2020, pour retomber progressivement à $0.9 \%$ en 2030. L'indicateur des ressources naturelles est assez lourd d'enseignements. Il nous indique que le taux de croissance des pays à fortes ressources naturelles est en partie un taux d'altération de leurs ressources non renouvelables et qu'à long terme ces mêmes pays connaissent une chute brutale de leur croissance, précisément parce qu'ils n'investissent pas suffisamment dans leur développement, l'innovation et leur compétitivité. La chute progressive du cours du pétrole depuis mi-2014 (après un prolongement prévisible jusqu'en 2016) ne fait que confirmer cette appréciation. En conclusion, c'est surtout à partir de 2020 que la faiblesse des structures financières des pays du sud de la méditerranée constituera un point critique de non-retour. En ce qui concerne l'Algérie, la phase tampon sera certes peut-être plus longue en raison des ressources non négligeables dont elle dispose encore pour plusieurs années, mais sa compétitivité n'en est pas moins en question pour l'avenir ${ }^{3}$. Les composantes de la compétitivité des pays selon le $\mathrm{WEF}^{4}$ sont généralement regroupées dans les 12 catégories

\footnotetext{
${ }^{1}$ Dit autrement, $c^{\prime}$ est de la croissance à court terme que naîtra le contrechoc de la crise, et des visions à long terme que surgira le développement.

2 cf. Rapport du World Economic Forum - 2011, « Scenarios on the future of the Mediterranean region - 2030 »

${ }^{3}$ L'Algérie est arrivée aux limites ultimes de son économie de la rente fondée essentiellement sur l'exploitation d'énergies fossiles et de minerais. La consommation nationale d'énergie primaire est passée de 37.4 Mtep en 2001 à 53.3 Mtep en 2013 ; tandis que dans le même temps les exportations sont passées de 104.7 Mtep à 101.5 Mtep. Les tendances inversées de ces deux courbes se rencontreront au mieux en 2019-2020. En 2013, la production d'électricité aura mobilisé plus de $40 \%$ de la consommation nationale de gaz naturel alors que la production nationale n'a que très peu évolué, passant de $74353 \mathrm{k}$ tep en 2001 à $77058 \mathrm{k}$ tep en 2013, soit une évolution de 3,63\%. Ajoutée à la tendance baissière des exportations de gaz naturel de 19,5 $\%$ depuis 2005, il semble que ces données ne soient pas favorables au maintien pérenne d'une économie nationale fondée sur la rente énergétique. Approximativement, le pays ne dispose plus au mieux (c'est-à-dire à tendances constantes) que de 5 ans pour renverser la tendance anxiogène de son économie. Ce laps de temps peut se réduire sous l'influence des innovations en matière d'énergies renouvelables ou des politiques énergétiques extérieures.

${ }^{4}$ WEF « La compétitivité des pays arabes », Rapport 2010.
} 
suivantes (l'ensemble des institutions, des politiques et des facteurs qui déterminent le niveau de productivité d'un pays) :

1. Institutions : éléments indispensables à la compétitivité dans la mesure où elles déterminent le cadre juridique et administratif d'interaction entre les personnes, les entreprises et les pouvoirs publics pour générer des richesses. Par exemple, on inclut dans cette catégorie des droits de propriété clairement définis et exercés, une administration publique efficace et transparente, un système judiciaire impartial et indépendant, l'assurance d'une sécurité physique ainsi que des normes de gouvernance d'entreprise très strictes.

2. Infrastructure : Élément essentiel à l'activité économique pour un certain nombre de raisons. L'infrastructure de transport revêt une importance capitale dans l'acheminement rapide et à faible coût des marchandises sur les marchés, les services de télécommunication assurent une communication efficace et l'approvisionnement en électricité permet une production fluide et ininterrompue.

3. Stabilité macro-économique : composante importante dont l'absence rend le fonctionnement des entreprises difficile. L'inflation limite la capacité des entreprises à planifier et à investir, et les climats de lassitude fiscale prolongée, une dette publique élevée ou un système financier inefficace peuvent donner lieu à une diminution des fonds pour l'investissement ainsi qu'une augmentation des taux d'intérêt.

4. Santé et enseignement primaire : composante cruciale, en effet, une maind'œuvre en bonne santé et scolarisée est bien plus disposée à exploiter l'intégralité de son potentiel.

5. Il est impossible pour les pays de gravir les échelons du développement sans investir dans l'enseignement supérieur et la formation, eu égard aux exigences de compétences qu'impliquent les processus de production et d'élaboration de produits plus complexes.

6. Une concurrence vigoureuse est un facteur de rentabilité et d'innovation dès lors qu'elle exclut du marché les entreprises inefficaces et facilite l'entrée de nouvelles activités.

7. Efficience du marché du travail : est un élément nécessaire pour garantir une utilisation optimale des talents au sein d'une économie. Un marché du travail souple, assorti de structures d'encouragement méritocratiques et dépourvu de pratiques discriminatoires à l'encontre des groupes sociétaux est le mieux placé pour contribuer à la compétitivité.

8. L'attention s'est dernièrement fortement cristallisée sur le fonctionnement des marchés financiers. Le développement des marchés financiers englobe deux facteurs primordiaux d'accroissement de la compétitivité : la rentabilité du système financier comme source de financements destinés aux entreprises et la stabilité ainsi que la fiabilité du système financier.

9. Ouverture à la technologie : traduit l'aptitude d'un pays à adopter les technologies les plus récentes et à les utiliser pour accroître la productivité. 
L'adoption des technologies et l'innovation technologique n'ont pas les mêmes effets sur la compétitivité. L'adoption des technologies permet d'accroître la productivité des processus existants, tandis que l'innovation repousse les frontières technologiques. En grande partie, l'effet d'amélioration de la productivité, en particulier sur les marchés émergents encore éloignés des frontières technologiques, peut être mis à profit moyennant l'adoption de technologies extérieures.

10. Taille du marché : cette composante est prise en compte en raison des marchés de grande envergure qui, considérés comme des marchés intérieurs et élargis par les marchés internationaux, permettent aux entreprises de réaliser des économies d'échelle.

11. Sophistication des entreprises : joue un rôle en faveur de la productivité. La présence de clusters accentue la rentabilité de nombreux processus au sein des entreprises, tandis que les activités de type marketing et distribution augmentent la productivité par le truchement d'une hausse de la valeur des produits et des services.

12. Comme indiqué plus haut, la composante d'Innovation peut repousser les frontières technologiques. Les entreprises des économies développées peuvent uniquement maintenir les niveaux de salaire élevés dans leur pays en déplaçant les frontières technologiques vers l'extérieur ; aussi se doivent-elles d'élaborer des produits et des services de pointe et/ou d'employer des processus uniques (distinctifs).

Les piliers essentiels pour les économies dont le développement est tiré par les dits «facteurs » reposent sur des paramètres de base (institutions, infrastructure, stabilité macroéconomique, santé et enseignement primaire). Les piliers essentiels pour les économies dont le développement est tiré par l'efficience reposent sur des sources d'efficience (enseignement supérieur et formation, efficience des marchés des produits, développement des marchés financiers, ouverture à la technologie, taille du marché, concurrence vigoureuse). Les piliers essentiels pour les économies dont le développement est tiré par l'innovation reposent sur des sources d'innovation et de sophistication (sophistication des entreprises et l'innovation). Au demeurant, ajoutons notamment que pour assurer croissance et développement aux pays émergents, et particulièrement dans la région MENA, la Banque mondiale promeut le renforcement des 4 piliers de l'économie de la connaissance par l'appui à des politiques publiques en matière d'institutions économiques et politiques (diminution des barrières douanières, état de droit, respect de la propriété intellectuelle etc.), d'éducation (alphabétisation, enseignement secondaire et supérieur...), de technologies de l'information et de la communication (e-development, e-administration, e-sourcing, e-procurement...), et des systèmes d'innovation ( $R \& D$, articles scientifiques et techniques publiés, dépôt de brevets et de marques...). La croissance par l'innovation que semble appelé de ses vœux le WEF n'est donc pas un programme spécifique en soi mais s'insère dans une politique générale plus large de soutien à la croissance en matière de gouvernance publique, de soutien au secteur privé et à la création d'emplois, de participation de la société civile et des femmes, de l'investissement dans l'éducation, de gestion des ressources. Comme on peut s'en rendre compte dans la figure 1, les pays du Maghreb se situent à un niveau de compétitivité relativement faible et ne font pas reposer leur développement sur l'innovation, 
à peine sur des facteurs d'efficience relativement maigres. Les défis de l'innovation sont donc intacts et nombre de mesures devront être engagées pour préparer un avenir posthydrocarbure pour l'Algérie ou post-tourisme pour d'autres pays du Maghreb.

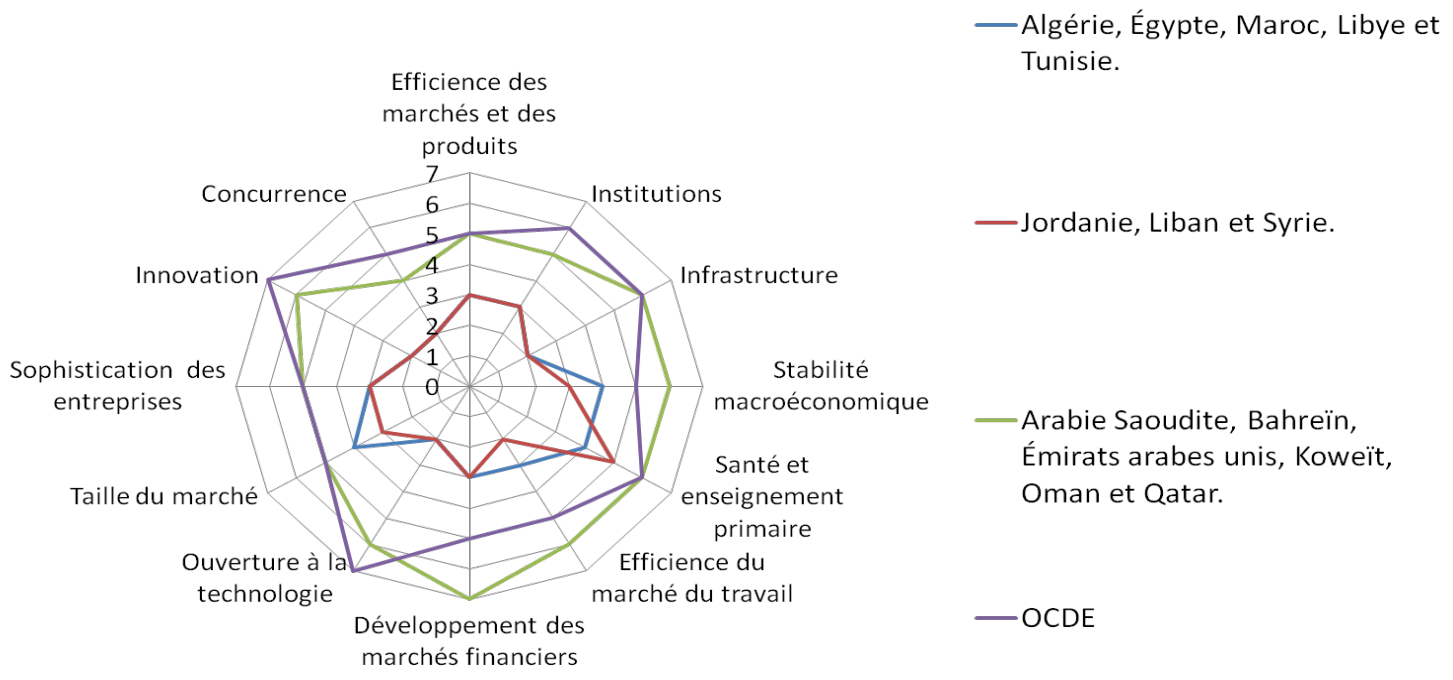

Figure 1 : Les pays du Maghreb entre facteurs et semi-efficience.

Avec un PIB de 160.3 milliards de dollars et plus de 173 milliards de réserves de change, l'Algérie n'est pas aux abois, même si elle reste à un niveau relativement faible si l'on ramène le PIB aux 35.4 millions d'habitants, ce qui nous ramène à un niveau 7 fois inférieur à celui de la Grèce et presque 3 fois en dessous de la Libye (en 2012). Les capacités stratégiques d'un pays sont une combinaison intelligente et forte de ressources, d'une économie de la connaissance et de compétences et savoir-faire. Or, si l'Algérie détient des ressources incontestables, elle n'est pas à ce jour en possession d'une réelle économie de la connaissance et de compétences fondamentales et compétitives. C'est un handicap majeur pour un rattrapage économique en période de crise internationale. L'une des voies possibles est le développement en réseau, en clustering, qui consiste à mettre sur pied tous les paramètres favorables aux investissements innovants localisés dans des lieux dédiés.

\section{Des opportunités de développement en réseau}

Une métamorphose des organisations mérite d'être questionnée, celle de la substitution de la notion réseau à celle de l'organisation associée notamment à des dynamiques de territoires.

\subsection{De la nature du réseau}

Si Froehlicher et al. (2001) tendaient à considérer que les compétences relationnelles des entreprises étaient devenues indispensables et qu'elles occasionnaient des métamorphoses profondes des organisations, le réseau est une forme d'organisation très ancienne remontant au moyen âge (Braudel, 1985). Il est resté pendant plusieurs siècles le modèle dominant caractérisant les transactions commerciales avant la révolution industrielle et l'apparition de la grande firme intégrée. Fernand Braudel (1985) a d'ailleurs montré l'existence de véritables " clusters » qui se sont développés en Europe et ont permis à certaines villes de bénéficier d'un rayonnement international singulier. Assens (2003) 
identifie trois phénomènes concomitants pouvant expliquer le regain d'intérêt du réseau en gestion à savoir, la mondialisation des échanges, les nouvelles technologies de l'information et la décentralisation des décisions. En effet, le réseau apparaît comme un mode d'organisation adapté à la mondialisation des échanges en permettant de relier des centres de décision autonomes (institutions, des banques, des États, des consommateurs, des clients, des actionnaires, etc.), entre différents marchés et territoires. Toutes les parties prenantes d'une entreprise peuvent être impliquées dans cette logique de ramification, à tel point que Callon (1991) substitue la notion de réseau à celle d'organisation. Le réseau est assimilé à une structure de transport qui offre la possibilité de réguler des flux de communications, ou des échanges de biens ou de personnes. Ce faisant, l'ensemble des réseaux dont la fonction principale réside dans le transport de ressources tangibles ou intangibles contribue à structurer le champ social, en permettant à des acteurs d'entretenir des liens affectifs, amicaux ou corporatistes, au même sens que Braudel (1985). Et c'est la raison pour laquelle ces réseaux de transport jouent un rôle majeur dans l'urbanisation et l'aménagement d'espace. Ce qui leur confère également une dimension économique par la valeur ajoutée qu'ils procurent aux membres.

Toutefois, même si le réseau offre une réalité différente, selon l'angle d'analyse, économique, sociologique, territorial, il conserve des caractéristiques essentielles et invariables. Ainsi, un réseau est toujours composé de nœuds, c'est-à-dire de points d'interconnexions capables d'émettre ou de recevoir des communications, capables de participer aux échanges ou de structurer les flux de transport. Ces nœuds sont reliés par des connexions qui traduisent la nature des échanges, leur périodicité, leur force, leur densité, etc., et ils occupent des positions qui sont susceptibles d'évoluer comme dans un réseau social ou qui demeurent figées comme dans un réseau technique ou territorial. Ces positions témoignent du rôle ou de la fonction assumés par chaque nœud à l'égard des autres membres du réseau.

Le concept de réseau repose ainsi sur quelques principes immuables l'autonomie des nœuds, l'interdépendance des nœuds et l'éloignement des nœuds (Assens, 1996). Le concept de réseau met donc l'accent sur l'articulation des relations entre les éléments d'une organisation, bien davantage que sur la nature des éléments eux-mêmes. La plupart des recherches en gestion sur le réseau découlent de ce constat. Elles focalisent l'analyse sur les processus d'interactions entre les membres d'un réseau, dans les alliances stratégiques interentreprises (Aliouat, 1996 ; Gulati 1998), dans les relations de confiance entre acteurs, dans les participations croisées entre différents conseils d'administration. Autant de questions qui portent sur la dimension ontologique des réseaux, analysée en fonction de la contingence de l'environnement et de la volonté des membres fondateurs. Car toute la difficulté de générer des coopérations réside à en faire converger les intérêts et les motivations parfois disparates des différents acteurs.

\subsection{De l'objet des réseaux}

Les motivations des acteurs sont très diverses à l'origine des réseaux. De nombreux auteurs se sont interrogés sur les causes d'apparition du réseau d'entreprises. Assens (2003) pose ainsi la question de savoir si le réseau est construit par une grande firme dont les activités se ramifient ou est-il le fruit d'interactions entre plusieurs petites sociétés ? Faut-il attribuer son origine à une décision intentionnelle ou à la nature d'un environnement 
spécifique ? Trouvons-nous des réseaux dans tous les secteurs d'activités ou dans des domaines particuliers?

\subsubsection{Le réseau comme réponse à la complexité des environnements}

Sur le plan économique, certains arbitrages conditionnent le choix d'une forme d'organisation pour les entreprises. Ces arbitrages s'opèrent selon un raisonnement décrit par la théorie des coûts de transactions (Williamson 1983) pour savoir s'il est plus rentable d'internaliser ou d'externaliser une activité, et par la théorie de l'interdépendance mutuelle des ressources (Pfeffer et Salancik, 1978), pour savoir comment contrôler les activités dont dépend l'avenir de l'entreprise. Ajoutées à la théorie des organisations, ces approches traitent l'entreprise comme un système ouvert en relation d'échanges avec leur environnement qu'elles aménagent, et dont elles tirent profit pour l'acquisition de leurs ressources et l'écoulement de leur production (Van de Ven, 1976). C'est précisément de ces échanges que naissent des relations de pouvoirs destinées à contraindre les décisions et les comportements d'autres organisations (Pfeffer, 1985). C'est le rôle du réseau dans la formation des alliances stratégiques qui s'impose ici.

La création d'un réseau répond effectivement à des contraintes extérieures poussant les firmes à se regrouper, mais également à une volonté de partenariat pour obtenir des avantages compétitifs. La théorie de l'avantage concurrentiel (Porter, 1986) considère que l'alliance permet d'optimiser les chaînes de valeur respectives des entreprises liées par l'accord. Dans le domaine des coûts de transactions, Jarillo (1988) présente le réseau comme une logique de cotraitance, alternative à la sous-traitance et à l'intégration. On parle aujourd'hui de colocalisation des processus de production. D'après ces travaux, dans certaines conditions d'environnement le réseau permet de diminuer les coûts de transaction. Le réseau d'entreprises émerge ainsi dans des industries de pointe ou dans des secteurs de service, caractérisés par des cycles de vie courts. Dans cet environnement particulier, les relations de confiance au sein du réseau facilitent la rapidité d'exécution et la flexibilité nécessaire aux évolutions technologiques et commerciales. Elles évitent le recours, sur un marché, à des intermédiaires nombreux pour négocier des contrats et, par conséquent, le risque d'opportunisme. Précisions que le contrôle de la dépendance des ressources ne résulte pas de facteurs internes (spécificité des actifs et compétences distinctives), ou externes (contrôle des ressources rares), mais de la capacité à préserver ou enrichir un capital relationnel (Dyer et Singh, 1998).

Selon Pfeffer et Salancik (1978), la relation au sein du réseau permet d'obtenir de l'information sur les activités des autres firmes qui influencent son propre résultat, d'introduire de façon récurrente des conventions de comportement avec les autres, d'obtenir le soutien d'une partie importante de son environnement auprès des fournisseurs, clients, concurrents et partenaires éventuels, de légitimer son rôle et sa fonction à l'égard des autres et d'instaurer un consensus social basé sur la confiance mutuelle. Dès lors, les interactions, dont le but est de stabiliser les termes des transactions, constituent " l'environnement négocié des firmes » (Pfeffer et Salancik, 1978).

\subsubsection{Le réseau comme choix stratégique}

Le réseau, dans la plupart des cas, est aussi une démarche stratégique pour atteindre un avantage compétitif de type coopératif (Dyer, Singh, 1998). Cette volonté collective peut 
être incarnée soit par une firme pilote et centrale qui fédère des firmes périphériques, soit par une communauté de PME qui souhaitent se regrouper dans une structure sans pilote (Gastaldi, 2000). À chaque fois, les fondateurs du réseau prennent conscience de l'intérêt mutuel d'une mise en relation, récurrente et durable, de leurs ressources et compétences. Cet intérêt repose sur le principe de la connivence entre acteurs et de la coopération entre entreprises.

$\mathrm{Vu}$ sous cet angle, le réseau offre alors des possibilités de business development, qui dépasse très largement la capacité individuelle de chacun des membres. Dans cette optique, les alliances au sein d'un réseau sont «protégées » des risques d'opportunisme de l'un des partenaires, à cause des barrières à l'entrée et des barrières à la sortie, érigées à travers les règles communes d'échange (Noteboom, 2006).

Dans le réseau, les alliances sont donc récurrentes, tout en impliquant davantage de parties prenantes (Gulati 1998). Dans ces conditions, les membres du réseau peuvent réaliser des économies d'échelle, partager des risques financiers ou innover par une fertilisation croisée des méthodes en recherche et développement (Assens, Baroncelli et Froehlicher, 2002). Cette vision économique du réseau est complétée par une approche sociologique, celle-ci explique que les alliances sont sous-tendues sur le plan social par une logique de coopération entre acteurs au sein des entreprises membres du réseau (Granovetter, 1985). Il précise que cette coopération est d'autant plus efficace que les acteurs sont encastrés dans une même communauté, partageant un code de conduite, ou un code de l'honneur les protégeant mutuellement des comportements déviants.

D'après ce constat, le champ des conventions sociales est susceptible de réguler les transactions économiques de façon beaucoup plus fine que le simple arbitrage par les prix. Granovetter (1985) met ainsi l'accent sur la force des "liens faibles », liens amicaux ou affectifs noués entre les acteurs, pour soutenir les "liens forts », liens marchands ou financiers noués entre les entreprises. Par ailleurs, dans une politique d'émergence d'écosystèmes d'affaires, tous les acteurs de l'économie, de la formation, de la recherche,....agissent de concert. Et permettent des actions de coévolution. De co-innovation. L'idée est de faire en sorte que des ensembles d'activités se construisent sur un territoire précis pour répondre à des marchés donnés qui se réinventent sans cesse. Le moteur d'une économie pérenne $c^{\prime}$ est d'abord de développer une activité pour répondre à un besoin existant. Puis cette activité engendre à son tour une multitude de besoins induits, susceptibles de susciter à leur tour de nouvelles opportunités commerciales. Cette logique n'a pas d'autres limites, en théorie, que celles de la créativité humaine...et donc de l'innovation.

On part d'une logique de marché, d'une approche client. Pas de ressources non renouvelables à transformer coûte que coûte. C'est la grande différence avec les logiques industrielles dans lesquelles un pays fondé sur la rente comme l'Algérie investit depuis 50 ans. C'est aussi le travers des entreprises privées en Algérie. Elles ne cessent de vouloir trouver des débouchés industriels aval à leur production amont sans se soucier des besoins réels du marché. Investissant dans des secteurs avals saturés et détruisant ainsi un tissu industriel local utile à des équilibres sociaux. Comme l'indique James Moore (1996), un écosystème d'affaires nait de l'interaction entre tous les organismes du monde des affaires : des entreprises et des individus. Cet écosystème d'affaires va produire des biens et des services en apportant de la valeur aux clients, qui font eux-mêmes partie de cet écosystème. 
Les membres vont inclure les fournisseurs, les producteurs, les concurrents et autres parties prenantes comme les organismes de formation ou de recherche. À travers le temps, ils vont faire co-évoluer leurs compétences et leurs rôles, et vont tendre à s'aligner eux-mêmes sur la direction d'une ou de plusieurs entreprises centrales (publiques ou privées). Ces entreprises vont détenir un rôle de leader qui peut évoluer à travers le temps mais la fonction d'un leader de l'écosystème sera d'apporter de la valeur à la communauté car il va engager les membres à agir en partageant une vision pour adapter leurs investissements et trouver des rôles d'appui mutuels. L'État devient un facilitateur de partenariats et d'alliances stratégiques entre entreprises privées ou publiques, amont et aval, Recherche/formation et entreprises,....Les relations de partenariats et d'alliances se démultiplient naturellement dans ces écosystèmes d'affaires régulés. Ensuite, le revenu final de l'opération sera partagé entre les différentes parties prenantes composant la chaîne des opérations. Les partenariats peuvent concerner tout à la fois la mise en commun de ressources ou le partage de compétences et ainsi entraîner un partage de risque global.

\subsection{Du mode de fonctionnement du réseau d'entreprises}

Le réseau possède des propriétés de fluidité par la polyvalence des configurations et de stabilité par la solidité de la trame relationnelle. La difficulté consiste à réguler harmonieusement cette dualité fragile de l'intérieur (conventions, pilote) ou de l'extérieur (médiateur, marché). Il s'agit à présent de décrire et d'expliquer les processus de régulation qui vont guider et coordonner les comportements au sein du réseau.

\subsubsection{Classification des réseaux d'entreprises}

Il convient tout d'abord de s'interroger sur le mode de pilotage. En théorie, le réseau fonctionne soit sur un mode supervisé, avec un ou plusieurs pilotes, identifiés et actifs, soit sur un mode d'autodétermination en l'absence de pilote (Assens 2001). Cette distinction nous conduit à classer les formes de réseaux d'entreprises dans deux catégories : les réseaux en étoile (ou star) et les réseaux de type communautaire. Dans la structure en étoile (structure star), le pouvoir de décision est réparti de façon inégale entre les partenaires. L'un des membres occupe une position dominante qui lui permet de coordonner et réguler les connexions avec les autres membres. En revanche, dans le réseau communautaire la fonction de pilotage est distribuée entre les membres. Il s'agit d'une architecture où toutes les entreprises sont reliées, sans qu'aucune ne détienne un rôle de régulation privilégié (comme dans le cas d'un « district industriel » européen).

Les réseaux d'entreprises se distinguent aussi par la nature des mécanismes de régulation pour assurer la coordination au sein du réseau. Nous prenons deux exemples pour illustrer les différences entre les modes de régulations. Tout d'abord le cas des grandes multinationales européennes et américaines qui s'organisent en réseau afin d'obtenir davantage de souplesse de fonctionnement par rapport à l'intégration et davantage de sécurité par rapport au marché. Ici les mécanismes de régulation sont surtout économiques. L'établissement de contrats, l'élaboration en commun de procédures et la négociation des prix permettent aux membres d'ajuster mutuellement les coûts d'approvisionnement, de production ou de diffusion. Dans ces conditions, le réseau star devient un moyen de diminuer les coûts de transactions pour l'ensemble des partenaires et pas seulement pour le pilote en position de commanditaire ou de coordinateur. Le réseau en étoile est alors assimilé 
à un système d'échanges, capable de planifier et de réagir comme une grande entreprise intégrée et dont l'efficacité interne serait comparable aux mécanismes de marché.

\subsubsection{Pour un modèle composite des réseaux}

Le réseau de firmes (business network) intrigue les chercheurs anglo-saxons qui considèrent qu'il s'agit d'une forme d'organisation transitoire et non permanente, au caractère hybride: une sorte de quasi-marché ou de quasi-hiérarchie (Williamson, 1983). Dans la littérature italienne à l'inverse, les chercheurs sont davantage convaincus de l'importance des réseaux dans la structuration de l'économie, même si la connaissance demeure souvent factuelle et difficile à modéliser. Pour de nombreux auteurs comme Callon (1991), Aliouat (1996), Dyer et Singh (1998), ou Assens (2002), cette difficulté à saisir l'essence du réseau provient du changement de paradigme requis dans l'observation des entreprises. Pour comprendre le fonctionnement d'un réseau, il convient d'étudier les relations entre les firmes plutôt que de se focaliser sur la nature des firmes elles-mêmes, que ce soit au niveau des ressources rares (Wernerfelt, 1984) ou des compétences clés (Barney 1991), ou que ce soit au niveau des frontières traditionnelles de l'organisation sur le plan patrimonial ou managérial, frontières jugées inopérantes selon Weiss (1994).

À travers les relations entre firmes, le réseau introduit une remise en cause importante des rôles et des statuts des intervenants : des alliances se nouent entre des concurrents et des fournisseurs deviennent clients. Ces phénomènes nous conduisent à envisager une nouvelle division du travail, entre des centres de décision dont les activités peuvent se chevaucher ou se compléter selon les circonstances. Avec la multiplication des ramifications internes et externes, l'organisation productive ne connaît plus de limites géographiques ou physiques, dans la mesure où les responsabilités et les enjeux s'étendent sur le plan territorial ou patrimonial. En effet, le réseau n'est pas forcément circonscrit par les limites d'un territoire, car il est toujours possible de coordonner un travail à distance grâce aux TIC. De même, les barrières du capital ne s'appliquent pas nécessairement, dans la mesure où les membres ne sont pas toujours intégrés dans une seule et même structure financière. Cependant, de nouvelles frontières sont instaurées, dans les conventions sociopolitiques (Granovetter, 1985) et dans l'harmonisation d'interface de communication (Curien 2000), par le partage d'intérêts communs.

\section{L'opportunité du « Clustering » en Algérie}

Conscient de l'intérêt historique, économique et social de la dynamique territoriale pour l'irruption ou le développement d'entreprises compétitives, nous assistons, depuis plusieurs années et partout à travers le monde, à l'instauration de politiques convergentes favorables à l'émergence de milieux innovants et durables. Ces politiques ont visé à construire des districts, des clusters, des parcs d'activités, des parcs technologiques ou plus récemment en France, des pôles de compétitivité. Ces effets permettent de lutter notamment contre le nomadisme industriel qui fragilise les équilibres économiques et sociaux. Ces choix sont souvent le fait d'entrepreneurs innovants sur des territoires appropriés et d'initiatives d'États soucieux de consolider leur compétitivité en matière d'innovation et leur capacité stratégique à long terme.

Cette politique de réseau au sens large consiste à mettre sur pied tous les paramètres favorables aux co-investissements innovants localisés dans des lieux dédiés. La question que 
pose notre travail est donc de savoir de quel degré de réseau et de " clustering » dispose l'Algérie pour s'engager dans une compétition mondiale?

\subsection{La formation des réseaux d'innovation territoriaux}

Les travaux de Marshall en 1891 sur les districts industriels, Porter (1998, 2000), ou encore Saxenian (1994) construisent les premières bases pilotes à propos du phénomène nouveau des logiques de réseaux d'innovation en analysant le fonctionnement et la gouvernance des réseaux qui se forment entre des entreprises et des institutions géographiquement proches. Ces dernières sont interdépendantes et liées par des métiers, des technologies et des savoir-faire communs. Ce sont des « clusters » dont la particularité est de générer un avantage compétitif significatif pour la nation toute entière. Cette structure de cluster marque un tournant dans nos logiques économiques et stratégiques, et va servir d'instrument de conversion des systèmes industriels classiques existants vers des modèles réticulaires et pluri-compétents fondés sur des synergies locales entre des organismes de recherche, de formation et des entreprises.

Les réseaux d'innovation technologique renvoient le plus souvent à des exemples réussis de territoires et de projets innovants au plan international et européen, à l'image des clusters (Porter, 1998) et des districts industriels (Marshall, 1910 ; Becattini, 1990). La proximité géographique, institutionnelle et organisationnelle de ces réseaux a d'abord été étudiée en économie spatiale dont les travaux abordent les milieux innovateurs à travers leurs relations localisées et coordonnées comme fondement principal de la compétitivité territoriale (Becattini et Rullani, 1995). L'économie géographique s'est emparée également du sujet pour mettre davantage en exergue la dynamique de proximité en opposant forces d'agglomération et effets de dispersion.

Les ancrages territoriaux, les trajectoires d'évolution, la path dependency, ou encore les investissements de proximité et la fidélisation à un territoire par les coûts de sortie prohibitifs s'inscrivent dans ces courants théoriques (Colletis et al., 1997). La sociologie ou les sciences de gestion ont cherché ensuite à enrichir l'analyse des formes de coordination au sein de ces relations localisées en ne se limitant plus exclusivement aux aspects matériels de la proximité spatiale afin de mieux comprendre soit la structure des interactions entre individus encastrés (Granovetter, 1985) soit l'intensité stratégique de tels réseaux pour la compétitivité des entreprises (Porter, 1998). Notre travail, focalisé à la fois sur la nature des réseaux et la dynamique d'acteurs/projets au sein d'un territoire donné, s'inscrit dans le prolongement de ces approches (Cooke et Piccaluga, 2005). La notion de réseaux d'innovation à laquelle nous faisons référence implique dans un premier temps d'opérer une distinction avec les réseaux d'entreprises engagées dans un processus d'innovation en commun, et dans un second temps de proposer une définition du réseau d'innovation territorial et de ses variantes. Nous confrontons ensuite ces réseaux d'innovation territoriaux à la question du choix de clustering pour les opérateurs institutionnels et économiques algériens.

\subsubsection{Du réseau d'entreprises aux réseaux d'innovation territoriaux}

Les réseaux d'entreprises sont construits autour d'une firme pivot ou d'un centre stratégique qui pilote un ensemble d'entreprises satellites. La position de la firme pivot est généralement dominante et cette domination est de nature économique ou technologique. 
Lorsque ces réseaux se structurent autour de projets d'innovation, on note que ces formes d'alliances stratégiques permettent d'appliquer avec plus d'efficience les connaissances acquises au sein du réseau (Baden-Fuller et Grant, 2004).

Le réseau d'innovation se définit, quant à lui, comme un ensemble coordonné d'acteurs hétérogènes (entreprises concurrentes ou non, laboratoires privés et publics, fournisseurs, clients, organismes financiers...) qui réalisent collectivement la conception, l'élaboration, la fabrication et la diffusion d'une innovation (Loillier et Tellier, 2002 ; Puthod et ThevenardPuthod, 2006). Les réseaux d'innovation territoriaux comprennent une dimension de proximité géographique comme facteur incitatif de coopération et peuvent prendre différentes formes : les districts industriels (Becattini et Rullani, 1995), les Systèmes Productifs Locaux (SPL) (Carluer, 2005), les technopôles (Callon, 1991), les clusters (Porter, 1998), et les pôles de compétitivité (Aliouat, 2010). Ils se transforment et s'étendent pour intégrer de nouvelles ressources afin de créer leur avantage compétitif.

Les districts industriels prennent la forme de relations interentreprises encastrées dans des zones géographiques favorables. Ils permettent de bénéficier d'effets d'agglomération et d'économie d'échelle conformément à la tradition marshallienne. Les Systèmes Productifs Locaux, nés d'une loi d'Orientation et d'Aménagement du Territoire en 1995, sont comparables aux districts mais ne se limitent pas aux relations de sous-traitance, ni même à une seule branche d'activité. Les technopôles en France se définissent comme " des concentrations géographiques locales d'entreprises innovantes, situées à proximité de centres de recherche et de formation scientifiques, dans le but de former ensemble un micro système innovant »(Ruffieux, 1991, p.375). Les clusters sont des pôles de compétence régionaux qui intègrent toutes les phases de la chaine de valeur économique (conception, production, distribution). Ils sont spécialisés dans un domaine technique et susceptible de procurer à leurs membres un avantage compétitif mondial. Les pôles de compétitivité (dans le dispositif français de la loi de 2005) constituent quant à eux plus spécifiquement des réseaux de connaissances.

\subsubsection{De la spécificité des pôles de compétitivité}

Les pôles de compétitivité ont donc un périmètre ouvert à trois ensembles d'acteurs : des entreprises, des acteurs de la recherche et des pôles de formation supposés constituer un terrain favorable à l'innovation, notamment parce qu'ils offrent une opportunité réelle de développer en leur sein des processus d'innovation ouverts multipliant les liens entre acteurs (essaimages, octroi de licences, partenariats en $R \& D, \ldots$ ). À ce titre, ils contribuent à accroître l'efficience et l'efficacité de la R\&D et de l'innovation (Chesbrough, 2003, 2006). On observe d'ailleurs que ces réseaux développent des formes de croissance nouvelles qui se propagent aux autres activités locales, notamment de service et de sous-traitance (Aliouat, 2010). Les différences majeures que nous observons entre les réseaux d'innovation classiques et les Pôles de compétitivité peuvent être résumées en cinq considérations. Une considération temporelle car les pôles de compétitivité (réseaux de connaissances) peuvent absorber des temps de coordination plus longs que les réseaux d'innovation (plus sensibles aux risques d'obsolescence); de gouvernance dans le sens où les réseaux d'innovation comprennent des firmes pivots alors que les pôles de compétitivité sont coordonnés et pilotés par des entrepreneurs institutionnels; une considération politique à l'origine des pôles de compétitivité tandis que les réseaux d'innovation classiques émergent sous l'impulsion d'entrepreneurs, c'est-à-dire bottom-up, une considération liée à leur mode de 
fonctionnement dans le sens où la volonté politique des pôles crée des interactions entre formation, recherche et entreprises, alors que les réseaux d'innovation classiques peuvent ne contenir que des entreprises. Contrairement aux districts et aux clusters, les pôles de compétitivité ne sont pas des unités émergentes dites "bottom-up ", mais plutôt des prolongements des SPL et des technopôles qui sont issus d'initiatives publiques («top down »).

\subsection{Quid des réseaux d'innovation en Algérie?}

En Algérie, le territoire régional ou local laisse émerger des initiatives entrepreneuriales qui sont parfois complètement dissociées d'une volonté politique nationale. D'Est en Ouest, les entrepreneurs innovants se saisissent de leur terroir pour en valoriser toutes les ressources. Toute la question est de savoir si l'on peut favoriser et inciter ces échanges relationnels dans un territoire donné, et par conséquent favoriser des pratiques de co-innovation en réseau impulsée par des acteurs institutionnels. Une comparaison avec des modèles étrangers nous éclaire sur l'intérêt des pôles de compétitivité et sur le degré de clustering à construire.

\subsubsection{Le réseau comme espoir de développement économique en Algérie}

Les clusters, les districts, les grappes, ou les pôles de compétitivité constituent un thème sur lequel un grand nombre $d^{\prime}$ acteurs économiques, institutionnels et politiques fondent des espérances de croissance, d'innovation et de projets entrepreneuriaux conséquents en Algérie. Les initiatives verticales, locales ou de réseaux horizontaux ont toutes le même objectif, favoriser l'essor industriel par la dynamisation de pôles d'excellence sur des domaines bien identifiés. Les secteurs technologiques concernés sont nombreux et présentent des potentiels de développement stratégiques (Energies, Banque, Tourisme, TIC, Logiciels, Télécommunications, Biotechnologies, Agriculture,...).

Ces orientations stratégiques requièrent des compétences multiples qu'il est utile d'articuler en réseau sur des bases collaboratives actives et entrepreneuriales (Intelligence économique, Gestion de la connaissance, Sciences de l'ingénieur, R\&D, RID, Recherche fondamentale, Incubateurs, Gestion de projets, Financement de projets, Capital-risque, Politiques incitatives,...). Le développement des pôles de compétitivité vise à tisser des axes autour d'acteurs locaux forts et responsables. Le principe général de ces pôles est de favoriser la coopération en matière de Recherche-Innovation-Développement (RID), sur un territoire donné, de trois types d'acteurs, jusqu'à présent insuffisamment en contact (les entreprises, les centres de formation, initiale ou continue, et les unités de recherche, publiques comme privées). Cette coopération doit s'effectuer autour d'un ou plusieurs projets communs, à caractère innovant et disposant de la taille nécessaire à une visibilité internationale. Le soutien des pouvoirs publics à ces collaborations par la distribution d'un certain nombre d'aides financières peut contribuer au fonctionnement de ces pôles et au financement de certains projets communs. Dans ce contexte, l'innovation apparaît comme le résultat d'un système complexe d'interactions entre une grande diversité d'organisations.

\subsubsection{Le réseau est-il une voie réaliste pour assurer le développement en Algérie ?}

$\mathrm{Vu}$ les principes de clustering précités, l'Algérie est-elle un pays qui favorise l'émergence de projets d'investissement, de start-up ou de pépinières d'entreprises nouvelles ? Permet-elle de consolider sa chaîne de valeur globale propice à l'amélioration de 
ses capacités d'innovation et de sa compétitivité au plan international ? Les rankings agrégés de la Banque Mondiale laissent entrevoir un déficit d'efforts depuis 2010, et observent encore peu de résultats significatifs pour la compétitivité comparée de l'Algérie (154ème pays en 2015 avec une perte de 9 places depuis 2011, 7 de 2014 à 2015). Le pays est encore 141ème dans le peloton de queue des nations pour la facilité à monter des affaires, dans l'amorçage de Start up ou d'affaires nouvelles. Par ailleurs, les ingrédients étatiques d'une compétitivité économique (droits de propriété, sécurisation des contrats, taxations, flexibilité législative, rapidité et agilité des procédures, protection des investisseurs et des parties prenantes, emplois,...) sont en perte de vitesse, et l'innovation ne semble pas non plus être au cœur d'une économie qui reste encore fondée sur la captation de rentes et non sur la création de richesses nouvelles. Les entrepreneurs et les porteurs de projets innovants ont à surmonter des contraintes d'environnement qui engendrent des coûts prohibitifs (importation de ressources matérielles transformées, importation de compétences et d'expertises, rigidité des procédures d'acquisition de ressources diverses,...).

Les indicateurs de coûts, de temps, de procédures et de dépôts de capital minimum nous permettent de conclure qu'en Algérie les procédures sont denses, rigides et longues, même si elles sont parfois moins coûteuses dans leur ensemble comparées à celles des pays Nord-africains et Moyen-Orientaux. Ces éléments de contreperformance ne sont pas propices à des dispositifs de clusters et nous ne pouvons les ignorer dans la mise en place de ces dispositifs. L'enregistrement de la propriété cumule aussi des indicateurs peu compétitifs de temps, de coût et de procédures. Les indicateurs d'obtention de crédits placent également l'Algérie dans les pays les moins incitatifs de l'entrepreneuriat privé, et ce de manière constante de 2008 à 2015. Le pays demeure ainsi en 2015 à la 171ème place au niveau mondial des pays facilitateurs de l'obtention de crédits (avec une perte de 42 places depuis 2013). Il est également 132ème des pays protecteurs des investisseurs minoritaires (perte de 59 places depuis 2010), 120ème des états de garantie contractuelle et de force exécutoire des contrats, 131ème des pays facilitateurs des activités transfrontalières et 176ème des états de taxation (perte de 17 places par rapport à 2012 après un gain de 4 places par rapport à 2010). Ces rankings nous indiquent que l'Algérie ne constitue pas encore un substrat favorable à l'émergence d'un entrepreneuriat dynamique et innovant qui permettrait au pays de substituer au modèle de captation de la rente fondée essentiellement sur l'exploitation des énergies fossiles, une économie entrepreneuriale créatrice de richesses en complément et en renforcement de la rente pétrolière. L'Algérie ne favorise pas encore l'émergence d'indicateurs facilitateurs de Start-up et de pépinières d'entreprises privées orientées vers l'innovation industrielle ou servicielle.

\subsubsection{Des volontés et des actes pour le développement des réseaux d'innovation en Algérie}

Les différentes formes de réseau évoquées sont des réseaux d'innovation assez proches, leur objectif est d'améliorer la compétitivité d'une nation pour l'économie de demain. Cependant, si l'Algérie doit encore favoriser, comme on vient de le voir, l'émergence de PME-PMI, dispose-t-elle des potentiels en formation et en recherche propres à ces réseaux d'innovation ? Il faut ici poser le postulat positif suivant : en 1962, l'Algérie comptait à peine quelques centaines d'étudiants dont $3 \%$ en sciences et technologie (deux écoles, une seule université sur Alger et ses deux annexes à Oran et Constantine). Aujourd'hui, le pays compte près de 1200000 étudiants, 25 Universités, 14 Centres 
universitaires, 16 Écoles et Instituts avec une très forte proportion de formations technologiques et ingénieures.

L'une des premières initiatives algériennes, le Cyberparc d'Alger, créé par décret $\mathrm{N}^{\circ}$ 04-275 du 05 septembre 2004, est assimilé à une grappe technologique s'insérant dans un périmètre urbanisable (le pôle urbain de Sidi Abdallah sous l'autorité du Ministère de l'aménagement $\mathrm{du}$ territoire et de l'environnement et du tourisme) où une surface est affectée au parc technologique (sous l'autorité du Ministère de la Poste et des Technologies de l'Information et de la Communication). Il est conçu au profit d'opérateurs nationaux et étrangers opérant dans le domaine des TIC. Le site entend comme n'importe quel cluster attirer des entreprises et des projets par des relais efficaces et attractifs. Une firme de dimension internationale dans le domaine des TIC est particulièrement recherchée. Dans la tradition des effets de clustering progressif, un deuxième parc technologique régional est prévu à Annaba.

Toutefois, il serait discutable de ne pas attendre les effets d'encombrement nécessaires sur le site d'Alger avant de concevoir le site d'Annaba. D'ailleurs, le Cyberparc d'Alger a une vocation nationale et même mondiale. Ce qui laisse entendre qu'il souhaite satisfaire des besoins qui dépassent la région d'Alger. La question reste cependant entière, le pays offre-t-il des dimensions de clustering attractifs et comment les acquérir ? À cette question, certains privilégient des réponses en termes de gouvernance pour attirer un maximum de projets et d'entreprise au sein de ces agglomérations innovantes ou industrielles. Les expériences réussies à l'international démontrent qu'il est essentiel de développer des modes de coopération au sein des régions qui puissent augmenter les externalités positives et favoriser l'innovation. L'objectif global est de favoriser, en effet, l'agglomération de compétences scientifiques, techniques et entrepreneuriales au sein de territoires qui n'ont pas forcément tous une longue tradition de partenariats. Une expérience algérienne est ici à mentionner qui pourrait servir de base à un clustering localisé.

Cette politique publique accompagne l'un des enjeux fondamentaux de tout pays et des entrepreneurs qui y exercent : celui d'assurer leur diversification au point de rencontre d'une opportunité (environnement économique favorable où les mutations industrielles, technologiques et financières observées ces dernières années ont induit une reconfiguration de la chaîne de valeur globale en faveur de l'avènement de nouveaux réseaux de soustraitance, de cotraitance et d'IDE des Pays Émergents) et d'une menace, celle d'un contexte mondial hyper compétitif et d'une raréfaction progressive des ressources énergétiques et naturelles. Ces mutations induisent une migration d'un profil d'exécutant vers un profil proactif.

\subsubsection{L'exemple français largement suivi en Algérie}

En France, et c'est un exemple assez largement suivi par l'Algérie dans le cadre de ses projets de parc d'activités, le soutien des pouvoirs publics à ces collaborations par la distribution d'un certain nombre d'aides financières contribue au fonctionnement de ces pôles et au financement de certains projets communs. En Algérie, le Fonds National d'Investissement, né de la restructuration de la Banque Algérienne de Développement en mars 2009, est comme en France doté d'un capital de 150 milliards de dinars (1,5 milliards $€$ ) auquel il faut ajouter d'éventuelles ressources additionnelles et des levées de fonds sur les marchés de capitaux avec ou sans émission de garantie du Trésor. Cette transformation 
marque un recentrage sur une approche de l'investissement par le marché et non plus sur le développement planifié par l'État. Cette forme de contribution reste cependant sous la forme de prêts, le plus souvent à des PME et ne prend pas la forme dédiée des aides courantes aux pôles de compétitivité, même si son montant correspond parfaitement à celui du Fonds Unique Interministériel français voué à 71 pôles de compétitivité.

Ainsi par exemple, le Cyberparc d'Alger est financé notamment, selon un décret du 22 novembre 2006, par un Fonds d'appui à l'investissement selon les dispositions de l'article 28 de l'ordonnance $\mathrm{n}^{\circ} 01-03$ du 20 août 2001 relative au développement de l'investissement et celles de l'article 227 de la loi 01-21 du 22 décembre 2001 portant loi de finances pour 2002. Toutefois, la constitution d'un fonds doit nécessairement poser la question de la gouvernance dans l'utilisation et la valorisation de ces ressources. L'autonomie des entrepreneurs institutionnels, qui sont des relais de l'investissement de développement public ou privé, est une clef de réussite comme nous le verrons ultérieurement. C'est leur pouvoir de décision, de contrôle et de validation des projets qui fera le succès du développement voulu par ce type de fonds.

\subsubsection{Les expériences des autres pays comme voie de benchmarking prometteuse}

Toutefois, s'il est vrai qu'il n'y a pas de capitalisme sans État, sachons que le succès des clusters ne repose pas exclusivement sur l'aide de l'État. Elle est certes forte en France, mais elle reste faible en Allemagne et ponctuelle aux USA. Ces deux derniers pays ayant par ailleurs des clusters à succès...et davantage qu'en France. Par extension, pour le cas de l'Algérie, il y a là matière à benchmarker un modèle intéressant.

La logique de cluster ou de grappe suppose une organisation industrielle en réseau à condition que la chaine de valeur globale des secteurs soit tant ou peu construite. Ce qui n'est pas le cas dans de nombreux secteurs en Algérie. Ceci explique d'ailleurs, que le pays exporte généralement ses matières premières à l'état brut (dans une logique de captation de rentes), alors qu'il pourrait maximiser ses recettes par l'instauration de pépinières d'entreprises qui transformeraient ces ressources naturelles et permettraient de maximiser la rente liée à ces ressources. Le gaz, le pétrole et les minerais, par exemple, ont des potentialités de transformation dont il serait judicieux d'exploiter les ressources en interne. À ce sujet, l'exemple du Dhahran Techno-Vallée en Arabie saoudite est très instructif. L'exemple de l'industrie automobile est aussi caractéristique des carences de chaîne de valeur globale en Algérie, carences qui constituent un frein aux IDE. Ce qui n'est pas le cas de l'Inde qui est présente à tous les échelons de la chaîne de valeur automobile, et de nombreux constructeurs ont fait le choix de ce pays pour s'approvisionner en équipement. En effet, ils y trouvent des capacités de design, d'ingénierie, et d'usinage très développées, une forte disponibilité d'ingénieurs à bas salaire et plus généralement une main d'œuvre qualifiée peu coûteuse et anglophone et surtout de réelles compétences en matière de TIC.

C'est donc toute la chaîne de valeur qui est à repenser et à structurer avant de pouvoir concourir sérieusement sur ce marché lucratif dans les pays émergents. Les effets de grappe (proches des districts industriels marshalliens ou des parcs d'activités italiens) sont de nature très diverses qu'il s'agisse d'entreprises informatiques en Irlande, d'entreprises de haute technologie à Bangalore (Inde), Soho pour l'industrie de production, Paris pour l'industrie de la mode, Detroit ou Turin pour l'automobile, Milan pour le textile et le cuir, Hollywood pour l'industrie du cinéma, ou même Las Vegas pour les jeux de hasard. La Silicon Valley y a 
ajouté des effets cluster nouveaux : les espérances de gains et de financement plus élevées, une plus forte demande, des taxes favorables, une concurrence stimulante et une configuration politique incitative. Il arrive que les grappes migrent vers des logiques de cluster. Les industries automobiles et des circuits intégrés de Détroit par exemple sont allés en Californie pour créer des structures d'innovation dans la perspective de rejoindre les avantages de la Silicon Valley. Que l'Algérie (et donc ses acteurs locaux) puisse concrètement aboutir à un horizon de quelques années, à concevoir et développer des produits et services technologiquement innovants et économiquement viables est une perspective que semblent soutenir d'ailleurs tant les acteurs privés que publics. Ainsi, de nombreux cas de clusters sont à benchmarker en Algérie dont les ressources et les configurations multiples appellent certainement des expériences elles-mêmes différenciées. Nous avons évoqué le Dhahran Techno-Valley dans le domaine des énergies, mais bien d'autres sont envisageables.

\section{Conclusion}

Dans un environnement hostile, dû à un défaut de régulation et de favorisation des activités entrepreneuriales, les coûts de transaction prohibitifs sont essentiellement générés par un contexte de risques exacerbés pour lesquels les entreprises consentent des efforts importants. Les actions en réseau sont alors d'une des formes possibles de limitation des coûts de transaction et de gains de performance face aux risques globaux.

Dans l'ensemble de notre analyse des clusters comme levier de développement pour un pays en transition, soulignons que les concepts de forums, de collaborations, de coopérations, de clubs professionnels, d'alliances, et de regroupements en tout genre ne sont pas nouveaux. Colbert en était un friand instigateur, le prix Nobel Ronald Coase relate des cas datant du moyen âge et Fernand Braudel nous livre des analyses succulentes au cœur d'une riche histoire du pourtour méditerranéen. Toutefois, leur implication dans des défis historiques de relance économique date surtout de la période post révolution industrielle et s'accentue depuis quelques décennies.

La création de pôles, grappes ou clusters initiée depuis quelques années par le gouvernement algérien va très certainement jouer un rôle majeur dans la dynamique des territoires à la sortie d'une succession de crises majeures financières ou géopolitiques. Accompagner et renforcer l'innovation collaborative afin de développer les meilleurs projets éligibles à des financements publics va probablement opérer un changement significatif dans le fonctionnement des pays et des Régions en transition, même s'il est encore trop tôt pour en anticiper tous les effets positifs. Finalement, nous identifions plusieurs facteurs clés pour réussir le développement de pôles et attirer des investisseurs et des porteurs de projets innovants. Premièrement, il apparaît important de définir et de communiquer un positionnement clair et différencié du cluster (sur la nature des recherches: soft, hard, immatériel, matériel ; sur le domaine technologique : nanotechnologies, biotechnologies, etc.) sur des axes capables de fédérer et motiver des grandes entreprises, des PME et des acteurs de la recherche et de la formation. Deuxièmement, il faut maîtriser des compétences fondamentales d'innovation au cœur des projets et des structures (ressources humaines, R\&D, management organisationnel, marketing, benchmarking, protection de la propriété intellectuelle, négociation contractuelle, Partenariats Public-Privé, dialogue de compétitivité et contrats de performance,...). Troisièmement, il apparaît nécessaire de construire un écosystème d'affaires, d'innovation et de financement incluant tout à la fois des acteurs 
publics et privés, y compris du capital risque et des business angels. Quatrièmement, il faut inscrire l'action d'un pôle dans la durée, avec des étapes successives et mesurables. Les pôles s'inscrivent en général dans un horizon de 5 ans, renouvelé pour 3 ans. C'est ainsi que les réseaux s'avèrent être des options fort utiles dans des contextes hostiles où les risques sont particulièrement prohibitifs.

\section{Bibliographie}

Aliouat, B. (dir.), (2010), Les pôles de compétitivité, Gouvernance et performance des réseaux d'innovation, Hermes Sciences Publishing, Lavoisier, Paris.

Aliouat, B., (1996), Les stratégies de coopération industrielle, Economica, Paris.

Aliouat, B., (2014a), L'entrepreneuriat algérien confronté à un manque de gouvernance inclusive : une approche conventionnaliste pour expliquer l'action de l'entrepreneur en milieu hostile, Revue Maghreb-Machrek, N²17, Avril, p.11-29.

Aliouat, B., (2014b), Développement algérien et réseaux d'innovation au Maghreb : enjeux, difficultés et propositions en préalable aux espaces économiques régionaux, in Sari C. et Mebtoul A., L'intégration économique maghrébine : un destin obligé ?, Editions L'harmattan, Paris.

Assens, C. (1996), « Du modèle bureaucratique au modèle organique : l'organisation en réseau », Flux, Cahiers du CNRS, $n^{\circ} 23$, p. 38-42.

Assens, C. (2001), "Stability and plasticity in self-organized networks », European Journal of Economic and Social Systems, vol. 14, n 4, p. 311-332.

Assens, C. (2003), Le réseau d'entreprises : vers une synthèse des connaissances, Management International 7 (4), p. 49-59.

Assens, C.; Baroncelli, A.; Froehlicher, T. (2002), "Cultiver un avantage technologique dans les réseaux : le cas de la Compagnie Générale des Eaux ", Gérer et Comprendre, Annales des Mines, $\mathrm{n}^{\circ} 68, \mathrm{p} .17-27$.

Baden-Fuller, Ch., Grant R.M., (2004), A Knowledge Accessing Theory of Strategic Alliances, Journal of Management Studies, January, Vol. 41 Issue 1, pp.61-84.

Barney J. (1991), "Firm Resources and Sustained Competitive Advantage", Journal of Management, vol. 17, n¹, pp. 99-120.

Becattini, G. (1990), "The Marshallian industrial district as a socio-economic notion", in Pyke F., Becattini G., et Sengenberger W. (eds) (1990), Industrial Districts and Inter-firm Co-operation in Italy, Genève: International Institute for Labor Studies, pp. 37-51.

Becattini, G., Rullani E., (1995), Système local et marché global. Le district industriel, in Rallet A. et Torre A. (eds), (1995), Economie industrielle et économie spatiale, Paris, Economica, 171-192.

Becker, W. \& Dietz J., (2004), R\&D Cooperation and Innovation Activities of Frms-Evidence for the German Manufacturing Industry. Research Policy, 33(2), pp. 209-223.

Braudel, F. (1985), La dynamique du capitalisme, Paris, Arthaud.

Callon, M., (1991), "Réseaux techno-économiques et irréversibilité", in R. Boyer et al., Figures de l'irréversibilité en économie, Paris, Editions EHESS.

Caloghirou, Y., Ioannides S., Vonortas N.S., (2003), Research Joint Ventures, Journal of Economic Surveys, 17 (4), pp.541-570.

Carluer, F., (2005), "Réseaux d'entreprises et territoires : une matrice d'analyse stratégique", Revue Management et Avenir, 2005/4, nº, pp.7-25.

Chesbrough, H.W. (2006), Open Innovation: a New Paradigm for Understanding Industrial Innovation, in Chesbrough, H.W., Vanhaverbeke, W. and West, J. (Eds.): Open Innovation: Researching a New Paradigm, Oxford University Press, Oxford. 
Chesbrough, H.W., (2003), Open Innovation, the new imperative for creating and profiting from Technology, Harvard Business School Press.

Colletis, G., Gilly J.-P., Pecqueur B., Perrat J., Zimmermann J.-B., (1997), "Firmes et territoires: entre nomadisme et ancrage", Revue Espaces et Sociétés, Décembre, n88/89, pp.115-138.

Cooke, P., Piccaluga A. (eds.), (2005), Regional Economies as Knowledge Laboratories, Edward Elgar.

Dachs, B., Ebersberger B., LÖÖF H., (2008), The Innovative Performance of Foreign-owned Enterprises in Small Open Economies, Journal of Technology Transfer 33, pp. 393-406.

De Faria, P., Lima F., Santos R., (2010), Cooperation in Innovation Activities: The Importance of Partners, Research Policy, 39(8), pp.1082-1092

Dyer, J., Singh H., (1998), "The Relational View: Cooperative Strategy and Sources of Interorganizational Competitive Advantage", Academy of Management Review, № 23 (4), pp. 660-679.

Froehlicher, Th., Khun A., Schmidt G. (dir.), (2001), Compétences relationnelles et métamorphoses des organisations, Editions Eska, Paris.

Gomes-Casseres, B., Hagedoorn J., Jaffe A.B., (2006), Do Alliances Promote Knowledge Flows? Journal of Financial Economics, Vol. 80, pp. 5-33.

Granovetter, M., (1985), "Economic Action and Social Structure: The Problem of Embeddedness", American Journal of Sociology, 91(3), pp.481-510.

Gulati, R. (1998) Alliances and Networks, Strategic Management Journal, 19 (4), 1998, pp.293-317

Jarrillo, J.C., (1998) « On strategic Networks », Strategic Management Journal, 9-1 pp 31-41

Loillier, T., Tellier A., (2002), "Innovations, coopérations et proximités, quelles configurations pour les réseaux innovateurs ?", Actes de la XIème conférence AIMS, Paris, juin.

López, R.A., (2008), Foreign Technology Licensing, Productivity, and Spillovers, World Development, Elsevier, vol. 36(4), April, pp. 560-574.

Marshall, A. (1910), Principles of Economics, 8th edition, Macmillan, London, First edition [1891] (London: Macmillan).

Moore, J., (1996), The Death of Competition - Leadership and Strategy in the Age of Business Ecosystems, Harper Business.

Mowery, D., Oxley J., Silverman B., (1998), Technological Overlap and Interfirm Cooperation: Implications for the Resource-based View of the Firm. Research Policy 27, pp.507-523.

Nijssen, B., O'Donnell G.M., Lettenmaier D., Lohmann D., Wood E.F., (2001), Predicting the Discharge of Global Rivers, J. Clim., 14, pp.3307-3323.

Nohria, N., Garcia-Pont, C. (1991), Global Strategic Linkages and Industry Structure, Strategic Management Journal, Special issue, Summer, 12, pp 105-124.

Nooteboom, B., (2006), Innovation, Learning and Cluster Dynamics, in Asheim B., Cooke P. et Martin R. (eds) (2006), Clusters and Regional Development, Critical reflections and explorations, Londres: Éd. Routledge, pp. 137-163.

Pfeffer, J., (1985), "A Ressource Dependence Perspective on Intercorporate Relations", Stanford University, Graduate School of Business, Working Paper n ${ }^{\circ} 794$.

Pfeffer, J., Salancik G.R., (1978), The External Control of Organizations: a Resource Dependence Perspective, New York, Harper \& Row Publishers.

Porter, M. (2000), "Location, Competition and Economic Development: Local Clusters in a Global Economy", Economic Development Quarterly, Vol. 14, No. 1, pp. 15-34.

Porter, M., (1986), L'avantage concurrentiel, Paris, InterEditions.

Porter, M., (1998), "Clusters and the New Economic of Competition", Harvard Business Review, vol. 76, n. 6, novembre-décembre, pp. 77-90. 
Stratégie de réseau : une source de développement et de performance pour les entreprises en situation de risques prohibitifs

Puthod, D., Thevenard-Puthod C. (2006), 'Coopération, tensions et conflit dans un réseau d'innovation construit autour d'une PME', Revue Française de Gestion, vol. 32, n 164, pp.181-204.

Rodrigues Bandeira, L.K., Amewokunu Y., Karuranga E., (2012), La Performance des Réseaux : Les Cas de Deux Réseaux Brésiliens, Revue Management International, Vol.4, N4, pp.85-102.

Ruffieux, B., (1991), Micro-système d'innovation et formes spatiales de développement industriel, in R. Arena et Al. (dir.), Traité d'économie industrielle, Economica, pp.373-382.

Saxenian, A., (1994), Regional Advantage, Culture and Competition in Silicon Valley and Route 128, Harvard University Press.

Van De Ven, A.H., (1976), "On the Nature, Formation and Maintenance of Relations Among Organizations", Academy of Management Review, n4 ${ }^{\circ}$, pp.24-36.

Weiss, S.E., (1987), Creating the GM-Toyota Joint Venture: a Case in Complex Negociation, The Columbia Journal of World Business, Vol.XXII, n², pp.23-37.

Wernerfelt B. (1984), A resource-based view of the firm, Strategic Management Journal 14, pp.4-12.

Williamson, O.E., (1975), Markets and Hierarchies: Analysis and Antitrust Implications, New York, Ed. Free Press. 